\title{
High seroprevalence of toxoplasma gondii and HIV-1 co-infection among drug users in Yunnan province, southwest China
}

\author{
Xin Chen ${ }^{1,4 \dagger}$, Mei Ye ${ }^{1,4 \dagger}$, Yan-Heng Zhou ${ }^{1}$, Feng-Liang $\mathrm{Liu}^{1}$, Lin $\mathrm{Duo}^{2}, \mathrm{Hong}^{3}{ }^{3}$, \\ Wei Pang ${ }^{1} \&$ Yong-Tang Zheng ${ }^{1 *}$
}

${ }^{1}$ Key Laboratory of Animal Models and Human Disease Mechanisms of the Chinese Academy of Sciences \& Yunnan Province, Kunming Institute of Zoology, Chinese Academy of Sciences, Kunming 650223, China;

${ }^{2}$ The Second People's Hospital of Yunnan Province, Kunming 650021, China;

${ }^{3}$ Yunnan Center for Disease Control and Prevention, Kunming 650022, China;

${ }^{4}$ Kunming College of Life Science, University of Chinese Academy of Sciences, Kunming 650204, China

Received March 14, 2016; accepted March 31, 2016; published online June 12, 2016

Citation: Chen, X., Ye, M., Zhou, Y.-H., Liu, F.L., Duo, L., Li, H., Pang, W., and Zheng, Y.T. (2016). High seroprevalence of toxoplasma gondii and HIV-1 co-infection among drug users in Yunnan province, southwest China. Sci China Life Sci 59, 857-859. doi: 10.1007/s11427-015-0358-y

Dear Editor,

Toxoplasma gondii was discovered more than 100 years ago and has a broad-spectrum of intermediate hosts in addition to its definitive host, felids. Although it does not cause symptomatic illness in most adults, it can lead to mental retardation in congenitally infected children and serious diseases in immunocompromised patients (Hill et al., 2005). The first report of $T$. gondii infection in China was published in Chinese in 1964; subsequently, many other cases have been carried out. Currently, most publications about the seroprevalence of $T$. gondii infection in Yunnan have examined infections in pets, domestic animals, and wild animals; however, few previously published studies have assessed human T. gondii infection in Yunnan province.

From March to December 2009, a voluntary cross-sectional survey was carried out among all drug users that our facility could follow in communities and detoxification centers in Qujing, Lincang, Zhaotong, Baoshan, and Dehong prefectures in Yunnan province, Southwest China. After obtaining written informed consent, an anonymous questionnaire-based interview was performed and basic socio-demographic information was acquired. Additionally,

$\dagger$ Contributed equally to this work

*Corresponding author (email: zhengyt@mail.kiz.ac.cn)
$5 \mathrm{~mL}$ venous blood was collected in vacuous tubes with added EDTA-2K. Plasma was extracted by centrifugation and stored at $-80^{\circ} \mathrm{C}$ for subsequent analyses. Anti- $T$. gondii IgG antibodies were screened with diagnostic enzyme-linked immunosorbent assay (ELISA) kits (Zhuhai S.E.Z Haitai Biological Pharmaceuticals Co., Guangzhou), and $T$. gondii IgG-positive tests were confirmed using another diagnostic ELISA kit (Tangshan INNOVITA Biotechnology Co., Tangshan). Anti-HIV antibodies were assessed using diagnostic colloidal gold kits (Alere Medical Co., Japan), and the HIV-positive tests were confirmed using diagnostic ELISA kits (Beijing Wantai Biological Pharmacy Enterprise Co., Beijing). Specimens with positive results in both assays were considered to be positive for $T$. gondii/HIV. All tests were performed according to the manufacturer's instructions.

Data obtained from questionnaires and previous laboratory tests were analyzed using the software package Statistical Package for Social Sciences (version 22.0; SPSS Inc., USA). Categorical variables were compared using $\chi^{2}$ tests; all tests were two tailed and differences between means with $P$-values less than 0.05 were considered to be significant.

A total of 1,414 drug users were recruited from the following five prefectures of Yunnan Province: Qujing $(n=151)$, Lincang $(n=166)$, Zhaotong $(n=295)$, Baoshan 
( $n=443$ ), and Dehong ( $n=359)$. The age of these participants ranged from 13 to 68 years old, with a median age of $31 \pm 10$ years old. Among these drug users, most were males of Han ethnicity, and approximately half were farmers, unmarried, and had more than six-years of school education. Notably, $22.2 \%$ of these individuals were minorities, including Achang, Bai, Zang, Dai, Deang, Hui, Jingpo, Lisu, Man, Mian, Miao, Tujia, Yi, and Zhuang (Table 1).

Among our cohort of 1,414 drug users, $31.6 \%$ were found to be seropositive for anti- $T$. gondii $\mathrm{IgG}$ antibodies, and the seroprevalence of $T$. gondii varied markedly in different stratified groups (Table 1). Drug users who were living in the China-Myanmar border region (Baoshan and Dehong) had a much higher percentage of $T$. gondii infection than those living in other prefectures $(P<0.001)$. Elder drug users had a higher risk of $T$. gondii infection than younger ones $(P=0.007)$, and a similar phenomenon could be observed among illiterate versus well-educated individuals $(P=0.015)$. Although the sample size $(n=62)$ of female drug users was small, the rate of infection with $T$. gondii among male drug users was significantly higher $(P=0.001)$. Moreover, T. gondii seroprevalence among minorities was significantly higher than in Han Chinese (49.5\% vs. $26.4 \%$, $P<0.001$ ), and was higher among farmers than those with other jobs or who were unemployed. Conversely, there were no significant differences between drug users who were unmarried, married, cohabitated, divorced, or widowed, suggesting that sexual intercourse was not the transmission route of $T$. gondii.

Furthermore, $24.2 \%(342 / 1,414)$ of all participants were found to be seropositive for HIV antibodies. The percentage of $T$. gondii infection among HIV-positive drug users was significantly lower than among HIV seronegative drug users (24.6\% vs. $33.9 \%, P=0.001$; Table 1 ).

Prior to 2008, the seroprevalence of $T$. gondii in China ranged from $0.4 \%$ to $20.2 \%$ in blood donors, from $17.3 \%$ to $21.8 \%$ in intravenous drug users, and from $0.5 \%$ to $25.5 \%$ in pregnant women (Zhou et al., 2011). In this present

Table 1 Toxoplasma gondii infection characteristics among drug users in Yunnan, China ${ }^{\text {a) }}$

\begin{tabular}{|c|c|c|c|c|c|c|}
\hline \multirow{2}{*}{ Variable } & \multicolumn{2}{|c|}{ Total } & \multicolumn{2}{|c|}{ Toxoplasma seropositive } & \multirow{2}{*}{$\chi^{2}$ value } & \multirow{2}{*}{$P$-value } \\
\hline & $\mathrm{N}$ & $\%$ & $n$ & $\%$ & & \\
\hline Residence & & & & & 113.904 & $<0.001$ \\
\hline Qujing & 151 & 10.7 & 19 & 12.6 & & \\
\hline Zhaotong & 295 & 20.9 & 63 & 21.4 & & \\
\hline Baoshan & 443 & 31.3 & 167 & 37.7 & & \\
\hline Dehong & 359 & 25.4 & 173 & 48.2 & & \\
\hline Female & 62 & 4.4 & 8 & 12.9 & & \\
\hline Male & 1350 & 95.6 & 439 & 32.5 & & \\
\hline Age & & & & & 14.169 & 0.007 \\
\hline$\leqslant 25$ & 324 & 22.9 & 103 & 31.8 & & \\
\hline $26-30$ & 341 & 24.1 & 96 & 28.2 & & \\
\hline $31-35$ & 370 & 26.2 & 103 & 27.8 & & \\
\hline $36-40$ & 227 & 16.1 & 81 & 35.7 & & \\
\hline$\geqslant 41$ & 151 & 10.7 & 64 & 42.4 & & \\
\hline Han & 1098 & 77.8 & 290 & 26.4 & & \\
\hline Minority $^{*}$ & 313 & 22.2 & 155 & 49.5 & & \\
\hline Occupation & & & & & 63.097 & $<0.001$ \\
\hline Jobless & 513 & 37.8 & 106 & 20.7 & & \\
\hline Farmer & 632 & 46.6 & 268 & 42.4 & & \\
\hline Others ${ }^{* *}$ & 212 & 15.6 & 60 & 28.3 & & \\
\hline Marital status & & & & & 1.210 & 0.751 \\
\hline Unmarried & 627 & 44.3 & 189 & 30.1 & & \\
\hline Married & 564 & 39.9 & 184 & 32.6 & & \\
\hline Cohabitated & 55 & 3.9 & 19 & 34.5 & & \\
\hline Divorced or widowed & 168 & 11.9 & 55 & 32.7 & & \\
\hline Education & & & & & 10.497 & 0.015 \\
\hline None & 100 & 7.1 & 41 & 41.0 & & \\
\hline $1-5$ years & 508 & 36.0 & 176 & 34.6 & & \\
\hline $6-9$ years & 625 & 44.3 & 175 & 28.0 & & \\
\hline Negative & 1072 & 75.8 & 363 & 33.9 & & \\
\hline Positive & 342 & 24.2 & 84 & 24.6 & & \\
\hline
\end{tabular}

a) *, Achang, Bai, Zang, Dai, Deang, Hui, Jingpo, Lisu, Man, Mian, Miao, Tujia, Yi, Zhuang; **, Cook, Electrician, Cooker, Waiter, Government staff, Businessman, Nurse, Worker, Chauffeur, Builder, Hotel manager, Miner, Carpenter, Repairman, Salesman, Doctor. 
study, $31.6 \%$ of drug users were seropositive for $T$. gondii, which was much higher than the aforementioned results. As T. gondii could not transmit via blood or intercourse, the epidemic of $T$. gondii among drug users might suggest an increasing trend of $T$. gondii infection in China. Moreover, the seroprevalence of $T$. gondii in different prefectures of Yunnan province ranged from $12.6 \%$ to $48.2 \%$, which might reflect an epidemic status of $T$. gondii infection in some provinces of China. Together, our findings indicate that a nationwide survey is needed to monitor the exact seroprevalence of $T$. gondii infection in China.

As such a high percentage of drug users were infected with $T$. gondii, targeted interruptions were immediately needed to prevent toxoplasmosis and new infections. Based on our present study, well-educated drug users had a lower percentage of $T$. gondii infection, implying that education was an effective measure for reducing $T$. gondii infection. Additionally, education or public health programs should focus on the elderly male minorities who are doing farm-related jobs in the China-Myanmar border region, as they were the most badly impacted by $T$. gondii.

Drug users and HIV-positive patients have been considered to be prone to $T$. gondii infection because they have suppressed or damaged immune systems; however, this opinion has been the subject of debate. Previous studies have shown that the seroprevalence of $T$. gondii was not significantly different between HIV-positive and -negative illicit drug users in Iran (Alavi et al., 2013). In this present study, the rate of $T$. gondii infection among HIV-positive drug users was lower than among HIV-negative drug users, which was likely the reason that HIV infection showed no association with $T$. gondii infection. Moreover, few sites were sampled where the rates of HIV prevalence were high and those of $T$. gondii seroprevalence were low. Together, these findings indicated that immune status showed no association with $T$. gondii infection.

Although drug users and HIV-positive patients were not found to be prone to $T$. gondii infection, they were prone to toxoplasmosis if they had been infected by $T$. gondii. Previous studies showed that latent $T$. gondii infection would modulate immune responses during HIV infection, and showed a trend for reactivation among some HIV/T. gondii co-infected individuals; for example, in the form of toxoplasmic encephalitis (Beran et al., 2015; Kodym et al., 2015). In this present study, 447 drug users were found to be seropositive for $T$. gondii IgG antibodies, including 84 HIV/T. gondii co-infected individuals. Although it was difficult to follow-up previous participants, we attempted to the best of our ability to warn them about the risk of reactivation of latent $T$. gondii infection.

In this present study, although our findings were drawn from a large sample size, there were two main limitations. First, no factors about diet culture were included in the questionnaire. The authors knew that diet culture was a key factor in studies of $T$. gondii, and we had included this in our recent studies; unfortunately, in this present study, we could not provide this information because the primary purpose of sample collection was for HIV research, and re-interviewing these participants was impossible. These factors may not limit the main conclusions of this study, that a high prevalence of $T$. gondii infection exists in China and this demands more efforts to prevent it. Second, the findings drawn by this present study might be influenced by the partially answered questionnaires of some participants. Based on our statistical analyses, the seroprevalence of $T$. gondii showed no significant difference between the completely and partly answered questionnaires $(31.9 \%(n=1351)$ vs. $25.4 \%(n=63), P=0.278)$, indicating that this influence was likely negligible.

In conclusion, from our cross-sectional survey of 1,414 drug users in Yunnan Province, a much higher percentage of $T$. gondii infection was detected when compared with previously reported results from China. Further adequate studies and interruptions will be urgently needed to prevent the growing epidemic of $T$. gondii.

Compliance and ethics The author(s) declare that they have no conflict of interest.

Acknowledgements We thank all the volunteers who participated in this research. This work was supported by the National Natural Science Foundation of China (81271892, U1302224, U1202228), Key Scientific and Technological Program of China (2012ZX10001-006, 2012ZX10001-007). The funders had no role in study design, data collection and analysis, decision to publish, or preparation of the manuscript.

Ethical approval The protocol of this study was approved by the Ethics Committee of Kunming Institute of Zoology, Chinese Academy of Sciences (approval number: SWYX-2009021; approval date: January 7, 2009), and informed consent was obtained from all individual participants included in the study.

Alavi, S.M., Jamshidian, R., and Salmanzadeh, S. (2013). Comparative study on Toxoplasma serology among HIV positive and HIV negative illicit drug users in Ahvaz, Iran. Caspian J Intern Med 4, 781-784.

Beran, O., Kodym, P., Maly, M., Davidova, A., Reinvartova, G., Jilich, D., Holub, M., and Rozsypal, H. (2015). The effect of latent Toxoplasma gondii infection on the immune response in HIV-infected patients. Biomed Res Int 2015, 271842.

Hill, D.E., Chirukandoth, S., and Dubey, J. (2005). Biology and epidemiology of Toxoplasma gondii in man and animals. Anim Health Res Rev $6,41-61$.

Kodym, P., Malý, M., Beran, O., Jilich, D., Rozsypal, H., Machala, L., and Holub, M. (2015). Incidence, immunological and clinical characteristics of reactivation of latent Toxoplasma gondii infection in HIV-infected patients. Epidemiol infect 143: 600-607.

Zhou, P., Chen, Z., Li, H.L., Zheng, H., He, S., Lin, R.Q., and Zhu, X.Q. (2011). Toxoplasma gondii infection in humans in China. Parasit Vectors 4,165 .

Open Access This article is distributed under the terms of the Creative Commons Attribution License which permits any use, distribution, and reproduction in any medium, provided the original author(s) and source are credited. 